\title{
Esqueletopia e topografia da artéria celíaca no Lycalopex gymnocercus (Fischer, 1814)
}

\author{
Skeletopy and topography of the celiac artery in Lycalopex gymnocercus \\ (Fischer, 1814)
}

\author{
Leonel Felix Leão Neto, Erick Candiota Souza (D), Emanoelli Aparecida Rodrigues dos Santos, Marelise Moral Montana, \\ Amarílis Diaz Carvalho, Paulo Souza Junior (D) \\ Universidade Federal do Pampa (UNIPAMPA), Uruguaiana, RS, Brasil
}

\section{Resumo}

O Lycalopex gymnocercus (graxaim-do-campo) é um canídeo silvestre de médio porte que ocorre no cone sul. Tem dieta onívora e habita desde planícies descampadas até matas densas. A artéria celíaca é um dos principais ramos da aorta abdominal e possibilita a irrigação de importantes órgãos, tais como estômago, pâncreas, baço e duodeno. Objetivou-se estudar a topografia da artéria celíaca no $L$. gymnocercus, com ênfase em características comparativas e aplicadas. Para tal, foram dissecados, mensurados e radiografados 15 cadáveres de L. gymnocercus coletados mortos em rodovias. Foi registrada a posição de origem da artéria celíaca na aorta e sua esqueletopia em relação às vertebras. Também foi mensurada a distância entre as artérias celíaca e mesentérica cranial e verificada a correlação entre esta distância e o comprimento rostrosacral dos espécimes, bem como a existência de diferenças nesta distância entre os sexos. Em 67\% dos casos, a artéria celíaca foi o primeiro ramo visceral da aorta abdominal, emergindo caudalmente ao hiato aórtico. Em 33\%, a artéria celíaca originou-se cranialmente ao hiato aórtico, caracterizando uma origem torácica. Apresentou uma distância média da artéria mesentérica cranial de 6,66 \pm 1,85 mm, com valores mínimos e máximos de 4,63 e 10,83 $\mathrm{mm}$ respectivamente. Sua esqueletopia predominante foi ao nível da segunda vértebra lombar (L2), variando cranialmente até a primeira (L1). Houve correlação positiva moderada $(r=0,52)$ entre as distâncias das artérias celíaca e mesentérica cranial com o comprimento rostro-sacral dos indivíduos. Não houve diferença na distância entre as artérias quando comparados os sexos. 0 percentual elevado de indivíduos com origem da artéria celíaca cranialmente ao diafragma e a esqueletopia ao nível da segunda vértebra lombar foram as principais diferenças encontradas em relação aos carnívoros domésticos e pode ter implicação em medicina de animais silvestres.

Palavras-chave: Anatomia animal. Carnívoros silvestres. Esqueletopia. Sistema cardiovascular. 


\section{Abstract}

Lycalopex gymnocercus is a medium-sized wild canid that occurs in the southern cone. It has an omnivorous diet and inhabits from open plains to dense forests. The celiac artery is one of the main branches of the abdominal aorta and allows the irrigation of important organs. The aim of this study was to study the topography of the celiac artery in $\mathrm{L}$. gymnocercus, with emphasis on comparative and applied aspects. Thus, 15 cadavers of $\mathrm{L}$. gymnocercus, collected from roadkills, were dissected, measured and radiographed. The location of origin of the celiac artery in the aorta and its skelotopy in relation to the vertebrae was recorded. The distance between the celiac and mesenteric cranial arteries was measured and the correlation between this distance and the rostrum-sacral length of the specimens was verified, as well as the existence of differences in this distance between the sexes. In 67\% of cases, the celiac artery was the first visceral branch of the abdominal aorta, emerging caudally to the aortic hiatus. In 33\%, the celiac artery originated cranially to the aortic hiatus, characterizing a thoracic origin. The mean distance from the cranial mesenteric artery was $6.66 \pm 1.85 \mathrm{~mm}$, with minimum and maximum values of 4.63 and $10.83 \mathrm{~mm}$ respectively. The most common skeletopy system was at the level of the second lumbar vertebra (L2), varying cranially until the first (L1). There was a moderate positive correlation ( $r=0.52)$ between the distances from the celiac to the cranial mesenteric arteries with the individuals' rostrum-sacral lengths. There was no difference in the distance between the arteries when compared to the sexes. The high percentage of individuals with origin of the celiac artery cranially to the diaphragm and skeletopy at the level of the L2 vertebra were the main comparative differences to the domestic carnivores and may have implications in medicine of wild animals.

Keywords: Animal anatomy. Cardiovascular system. Skeletopy. Wild carnivorans.

\section{Introdução}

O Lycalopex gymnocercus (Fischer, 1814), conhecido como graxaim-do-campo ou raposa-dospampas, é um canídeo silvestre de porte médio (3 a $8 \mathrm{~kg}$ ), que apresenta pelagem avermelhada na região lateral da face e na extremidade distal dos membros, enegrecida em todo o dorso e cinza claro/ branco na face medial dos membros e face ventral da cabeça (Lucherini e Vidal, 2008).

Por ter uma dieta variada e boa adaptação ecológica (Lucherini et al., 2008), sua distribuição é ampla no sul da América do Sul. Existem relatos da ocorrência da espécie no leste/sudoeste da Bolívia, norte/nordeste da Argentina, sul do Brasil e Uruguai (Sillero-Zubiri et al., 2004; Di Bitetti et al., 2009). Habita tanto planícies pouco arborizadas como também florestas e regiões de mata densa. Consome principalmente pequenos vertebrados e frutos em situações oportunas (Canel et al., 2016). Pouco se sabe sobre o comportamento social da espécie, mas estudos apontam ser um animal monogâmico e de hábitos diurnos (Hunter, 2018).

Diante de levantamento bibliográfico recente, constatou-se que existem poucos estudos que abordam aspectos anatomorfológicos dos canídeos silvestres dos neotrópicos. Foram estudadas, por exemplo, características anatômicas do crânio e membro torácico do Cerdocyon thous e L. gymnocercus (Vaz et al., 2011; Pinheiro et al., 2014a; Souza Jr et al., 2014, 2015; Bubadué et al., 2016a,b), cone medular (Pinheiro et al., 2011), trato gastrointestinal (Ramos et al., 2011), algumas pesquisas acerca dos ramos do arco aórtico no $C$. thous (Engel etal., 2013; Lima et al., 2016), bem como morfometria do forame mandibular no Chrysocyon brachyurus (lobo-guará) (Souza Jr et al., 2016) e Speothos venaticus (cachorro-do-mato-vinagre) (Barroso et al., 2014). Existe, portanto, uma grande lacuna no conhecimento anatômico destes animais, importante para subsidiar a medicina veterinária de animais silvestres, bem como inferências ecológicas e evolutivas.

No tocante à anatomia do sistema cardiovascular, as informações são ainda mais escassas. Especificamente dentre os ramos viscerais da artéria aorta abdominal, a artéria celíaca é o primeiro ramo de origem ventral e imediatamente caudal ao hiato aórtico (Schummer et al., 1981; Dyce et al., 2010; König et al., 2016). Nos carnívoros domésticos, emite três ramos principais: artéria hepática, artéria gástrica esquerda e artéria esplênica (Getty, 1986; Evans, 1993).

O objetivo deste trabalho foi caracterizar a topografia da artéria celíaca do L. gymnocercus. 


\section{Material e métodos}

\section{Obtenção e preparo dos cadáveres}

Foram utilizados 15 cadáveres, sendo nove machos e seis fêmeas adultos, coletados nas rodovias da mesorregião sudoeste rio-grandense, sob autorização no 33667 do Instituo Brasileiro do Meio Ambiente e Recursos Renováveis/SISBIO e direcionados ao Laboratório de Anatomia Animal da Universidade Federal do Pampa (UNIPAMPA), campus Uruguaiana, RS, Brasil. Os espécimes foram injetados com solução de formaldeído puro e conservados imersos em solução de formaldeído a 10\%. Três cadáveres foram injetados com solução de látex (603 Bertoncini ${ }^{\circledR}$ ), misturada a um corante vermelho (corante líquido $\mathrm{Xadrez}^{\circledR}$ ), por meio de canulação da artéria aorta torácica. 0 acesso à artéria aorta foi realizado por abertura do sexto espaço intercostal. Apenas cadáveres com a artéria aorta e celíaca preservadas foram incluídos no estudo.

\section{Dissecção e mensurações}

Após remoção da pele, foram realizadas duas incisões: a primeira na linha alba, partindo do processo xifóide até a região púbica; a segunda transversalmente ao nível da última costela, em ambos os antímeros, partindo desde o processo transverso da primeira vértebra lombar até a linha ventral mediana. Após a localização da artéria aorta abdominal, foram dissecadas as artérias celíaca e mesentérica cranial.

Em seguida, para as mensurações, foi utilizado um paquímetro digital (ZAAS Precision, Amatools ${ }^{\circledR}$ ) para medir a distância entre os centros das origens das artérias celíaca e mesentérica cranial. Para a mensuração do comprimento rostro-sacral, utilizouse uma trena flexível, partindo desde a ponta do focinho até o ponto médio entre as tuberosidades sacrais.

\section{Esqueletopia}

Para a determinação da esqueletopia, os 15 cadáveres foram radiografados no setor de diagnóstico por imagem do Hospital Veterinário da
UNIPAMPA. Para demarcação da artéria celíaca, um alfinete foi inserido no centro da sua origem na aorta. Os animais foram posicionados em decúbito dorsal para obtenção de uma imagem em projeção ventrodorsal. A técnica utilizada foi a de chassi (cassete $24 \times 30 \mathrm{~cm}$ ) sobre a mesa, com aparelho de raios-X móvel (Aquilla Plus 300 Philips $^{\circledR}$ ) configurado a 47 KVp, $12 \mathrm{~mA}$ por 0,06 segundos.

\section{Estatística}

Os dados da distância entre as artérias celíaca e mesentérica cranial e o comprimento rostrosacral dos espécimes foram tabulados em planilhas no software BioEstat $5.3^{\circledR}$. Foi realizado o teste de correlação linear de Pearson entre a distância das artérias e o comprimento rostro-sacral. Assumiu-se que os valores de $r>0$ tinham correlação positiva, sendo fraca quando o valor fosse $0<r<0,5$, moderada quando $0,5 \leq \mathrm{r}<0,8$ e forte quando $0,8 \leq$ $r<1$. 0 teste de hipóteses $t$ de Student foi utilizado para comparar as médias das distâncias entre as artérias entre os sexos, considerando significativo quando $\mathrm{p}<0,05$.

\section{Resultados}

Em 67\% dos casos, a artéria celíaca originou-se da aorta abdominal e em 33\%, da aorta torácica. Em todos os animais a artéria celíaca teve origem como um tronco único, ventralmente na aorta. Quando teve origem na aorta torácica, emergiu de forma pré-diafragmática, atravessando pelo hiato aórtico e seguindo ventralmente para se ramificar.

A artéria celíaca estabeleceu relação topográfica caudal e lateralmente à esquerda da curvatura maior do estômago, cranialmente ao lobo esquerdo do pâncreas e medialmente em relação à glândula adrenal direita e fígado. No seu entorno, encontravase o plexo celíaco e celíacomesentérico.

Em 11 espécimes foi possível visualizar a esqueletopia por meio de radiografias. Em quatro indivíduos, as vértebras se apresentavam desmineralizadas em função do tempo prolongado de fixação em formaldeído e, portanto, não era possível uma nítida visualização da esqueletopia. Em 64\% dos casos, a artéria celíaca originou-se 
ao nível do corpo vertebral da segunda vértebra lombar (L2), em 18\% no espaço intervertebral entre L1e L2 e em 18\% ao nível do corpo vertebral de L1 (Figuras 1 e 2).
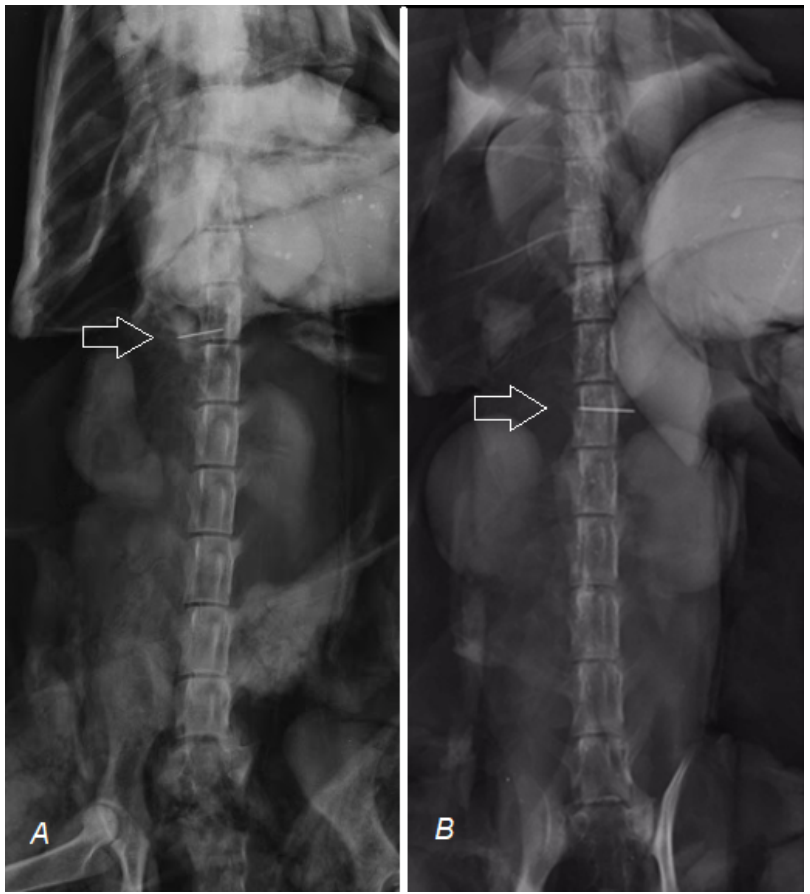

Figura 1 - Radiografia da região tóraco-abdominal de cadáver macho (A) e fêmea (B) de Lycalopex gymnocercus, em projeção ventrodorsal, evidenciando a esqueletopia da artéria celíaca ao nível da primeira e segunda vértebras lombares, respectivamente (marcação metálica - setas).

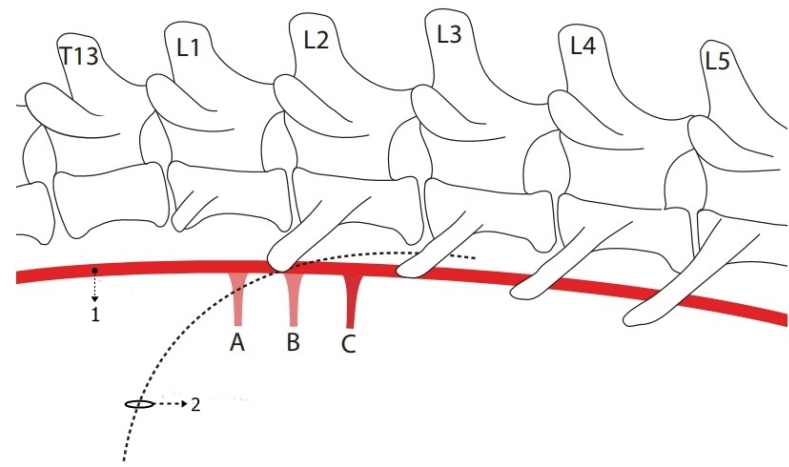

Figura 2 - Representação esquemática da esqueletopia da origem da artéria celíaca no Lycalopex gymnocercus. A: esqueletopia ao nível de L1; B: esqueletopia entre L1 - L2. C: esqueletopia ao nível de L2 (mais comum). Aorta (1); Diafragma (2).
A distância média entre as origens das artérias celíaca e mesentérica cranial foi de 6,66 $\pm 1,85 \mathrm{~mm}$, sendo a menor distância $4,63 \mathrm{~mm}$ e a maior 10,83 $\mathrm{mm}$. Nos machos, apresentou uma média de 6,42 \pm $1,20 \mathrm{~mm}$ e nas fêmeas 7,09 $\pm 2,80 \mathrm{~mm}$ (Figura 3). Não houve diferença significativa das médias das distâncias entre as artérias quando comparada entre os sexos $(p=0,52)$. Houve correlação positiva moderada $(r=0,52)$ entre as distâncias das artérias celíaca e mesentérica cranial com o comprimento rostro-sacral (Figura 4).

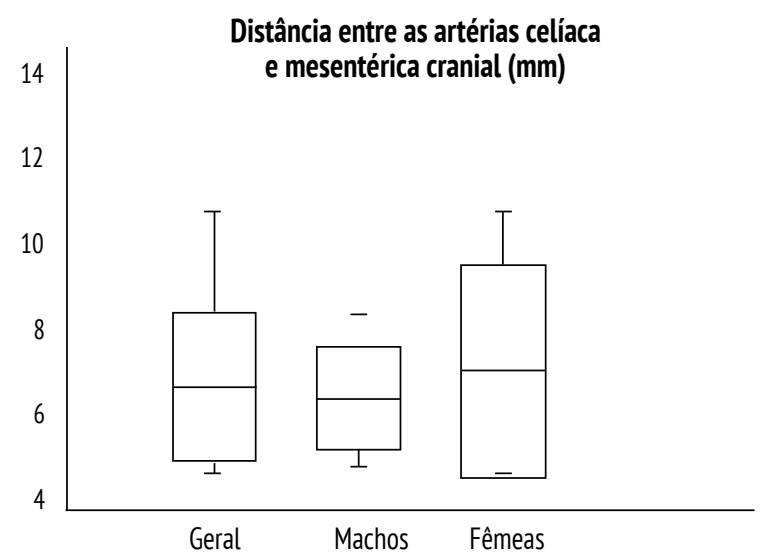

Figura 3 - Gráfico do tipo "box-plot" exibindo a média, desvio padrão, valores máximo e mínimo da distância entre as artérias celíaca e mesentérica cranial, em toda a amostra e separados por sexo.

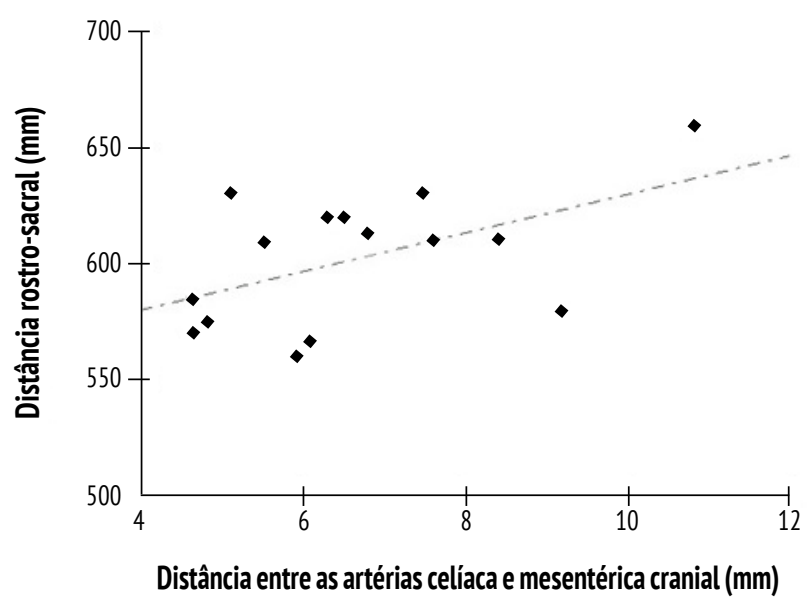

Figura 4 - Gráfico da correlação linear entre as distâncias das artérias celíaca e mesentérica cranial com o comprimento rostro-sacral demonstrando correlação positiva moderada e significativa $(p=0,0489)$ entre as variáveis. 


\section{Discussão}

Em 67\% dos espécimes de L. gymnocercus, a origem e topografia da artéria celíaca foram semelhantes às descritas para o cão doméstico (Schummer et al., 1981; Getty, 1986; Dyce et al., 2010; König et al., 2016), gato doméstico (Roza et al., 2009; Silva et al., 2012, 2013) e para a Jaguatirica (Leopardus pardalis) (Pinheiro et al., 2014b), ou seja, foi o primeiro ramo, de origem ventral, justaposto ao hiato aórtico, da aorta abdominal. Entretanto, não foram encontrados relatos da artéria celíaca proveniente da aorta torácica em espécies carnívoras, o que foi verificado em $1 / 3$ da amostra. Talvez, a alta ocorrência de origem torácica para a artéria celíaca no L. gymnocercus possa decorrer de um posicionamento mais caudal do diafragma nesta espécie. Em um relato, descreveu-se a ocorrência de um ramo pulmonar advindo da artéria celíaca em um cão doméstico (Roza et al., 2012).

Em espécies não carnívoras, a origem abdominal e ventral da artéria celíaca também ocorreu no Myocastor coypus (ratão-do-banhado) (Machado et al., 2002), Bradypus variegatus (preguiçacomum) (Gonçalez et al., 2003), coelhos Nova Zelândia (Abidu-Fiqueiredo et al., 2008), Didelphis albiventris (gamba-de-orelha-branca) (Culau et al., 2010), Mazama gouazoubira (veado-catingueira) (Amadori et al., 2012) e Galea psixii (preá) (Oliveira et al., 2017). Apenas foi descrita a existência da artéria celíaca com origem na aorta torácica em fetos bubalinos (Machado et al., 2000).

Nos espécimes de L. gymnocercus, a esqueletopia mais comum da artéria celíaca foi ao nível de L2 (64\%), sendo ao nível de L1 em 18\% e do espaço intervertebral de L1 e L2 em 18\%. No cão doméstico, a esqueletopia mais citada ocorre ao nível de L1 (Schummer et al., 1981; Getty, 1986; Evans, 1993). A esqueletopia ventral a L2 foi descrita em apenas $26,7 \%$ dos gatos domésticos (Silva et al., 2013).

Foi ilustrada a artéria celíaca localizada na região da última vértebra torácica (T18) em equinos (König et al., 2016), enquanto outros autores (Ashdown e Done, 2012) a referem como próxima a L2. No suíno, a esqueletopia da artéria celíaca ocorre ao nível de L1 (Getty, 1986; Gonçalez et al., 2003). Em um estudo realizado em coelhos Nova Zelândia, a maioria dos animais apresentou a artéria celíaca entre T13 e L1, ocorrendo em apenas um animal a aparição desta artéria ao nível de L1 (Abidu-Figueiredo et al., 2008). Porém, outro estudo apontou a origem ao nível de L1 em 62,5\% dos coelhos dissecados (Kuru, 2016). No Bradypus variegatus foi relatado que a artéria celíaca se situa ao nível de T12 e T13 (Campos et al., 2014). No Tamandua tetradactyla (Tamanduá-mirim), 75\% dos espécimes tinham origem da artéria celíaca ao nível de T16 (Macedo et al., 2013).

Existe uma escassez de dados sobre a distância entre as origens das artérias celíaca e mesentérica cranial. Descreveu-se em fetos bubalinos que a distância entre as respectivas artérias é de aproximadamente $3 \mathrm{~mm}$, variando entre 1,8 e $5 \mathrm{~mm}$ (Machado et al., 2000). Em humanos, foi de $12 \mathrm{~mm}$, variando entre 3 a $23 \mathrm{~mm}$ (Araujo-Neto et al., 2015). 0 valor médio encontrado no L. gymnocercus foi de 6,66 $\mathrm{mm}$, variando moderadamente de acordo com o comprimento do animal. A comparação deste valor com o de diferentes espécies, ou mesmo entre cães de diferentes raças, é difícil devido à variação de porte corporal e conformação dos animais.

\section{Conclusão}

Nos espécimes estudados de Lycalopex gymnocercus, a origem da artéria celíaca ocorreu na face ventral da artéria aorta, caudalmente ao hiato aórtico, embora em $1 / 3$ dos espécimes a origem verificada tenha sido na aorta torácica. A esqueletopia da artéria celíaca esteve predominantemente ao nível da segunda vértebra lombar (L2). A origem torácica e a esqueletopia ao nível de L2 foram as principais diferenças em relação aos carnívoros domésticos.

\section{Agradecimentos}

Os autores agradecem ao Programa de Desenvolvimento Acadêmico de 2017 (PDA), da Universidade Federal do Pampa. 


\section{Referências}

Abidu-Figueiredo M, Xavier-Silva B, Cardinot TM, Babinski MA, Chagas MA. Celiac artery in New Zealand rabbit: anatomical study of its origin and arrangement for experimental research and surgical practice. Pesq Vet Bras. 2008;28(5):237-40.

Amadori A, Birck AJ, Fidelpho AL, Guimarães GC, Peres JÁ, Souza RAM. Origem e distribuição da artéria celíaca em veado catingueiro (Mazama gouazoubira). Rev Cient Eletr Med Vet. 2012;10(19).

Araujo Neto SA, Franca HA, Mello Jr CF, Silva Neto EJ, Negromonte GR, Duarte CM, et al. Anatomical variations of the celiac trunk and hepatic arterial system: an analysis using multidetector computed tomography angiography. Radiol Bras. 2015;48(6):358-62.

Ashdown RR, Done SH. Atlas colorido de anatomia veterinária de equinos. São Paulo: Elsevier; 2012. 360 p.

Bubadué JM, Cáceres N, Carvalho RS, Meloro C. Ecogeographical variation in skull shape of SouthAmerican canids: abiotic or biotic processes? Evol Biol. 2016a;43(2):145-59.

Bubadué JM, Cáceres N, Carvalho RS, Sponchiado J, Passaro F, Saggese F, et al. Character displacement under influence of Bergmann's rule in Cerdocyon thous (Mammalia: Canidae). Hystrix It J Mamm. 2016b;27(2):83-90.

Campos GM. Análise morfológica do tronco celíaco e seus ramos no bicho-preguiça, Bradypus variegatus (SCHINZ, 1825) [dissertação]. Recife: Universidade Federal Rural de Pernambuco; 2014. 42 p.

Canel D, Scioscia NP, Denegri GM, Kittlein M. Dieta del zorro gris pampeano (Lycalopex gymnocercus) en la provincia de Buenos Aires. Mastozool Neotrop. 2016;23(2):359-70.

Culau POV, Reckziegel SH, Goltz LV, Araújo ACP. A artéria celíaca em Didelphis albiventris (gambá). Acta Sci Vet. 2010;38(2):121-5.

Di Bitetti MS, Di Blanco YE, Pereira JA, Paviolo A, Pírez IJ. Time partitioning favors the coexistence of sympatric crab-eating foxes (Cerdocyon thous) and pampas foxes (Lycalopex gymnocercus). J Mammal. 2009;90(2):479-90.

Barroso RMV, Santos ALQ, Kruger R, Milanezzii GP, Kurtt JS, Guimarães EC. Análise morfométrica do forame mandibular em Cachorro do Mato Vinagre (Speothos venaticus, LUND 1842). Pubvet. 2014;8(14):Art.1744.

Dyce KM, Sack WO, Wensing CJG. Tratado de anatomia veterinária. 4 ed. Cap 7. Rio de Janeiro: Elsevier; 2010. $856 \mathrm{p}$

Engel S, Maiochi RR, Birck AJ, Filadelpho AL, Guimarães GC. Origem e distribuição do arco aórtico do cachorrodo mato (Cerdocyon thous). Rev Cient Eletr Med Vet. 2013;11(21).

Evans HE. Heart and arteries. In: Evans He. Miller's anatomy of the dog. 3 ed. Philadelphia: WB Saunders; 1993. p. 586-682.

Getty R. Anatomia dos animais domésticos. 5 ed. Rio de janeiro: Guanabara Koogan; 1986. 2000 p.

Gonçalez PO, Carneiro e Silva FO, Severino RS, Drummond SS. Origens e ramificações da artéria celíaca em fetos de suínos (Sus scrofa domesticus Linnaeus, 1758) da linhagem "Rezende". Biosci J. 2003;19(1):87-95.

Hunter L. Field guide to carnivores of the world. 2 ed. London: Bloomsbury Wildlife; 2018. 272 p.

König HE, Ruberte J, Liebich HG. Órgãos do sistema cardiovascular. In: Anatomia dos animais domésticos: texto e atlas colorido. 6 ed. Porto Alegre: Artmed; 2016. p. 461-94.

Kuru N. Macroanatomic investigations of the course and distribution of the celiac artery in the New Zealand rabbit. Van Vet J. 2016;27(3):177-22.

Lima AR, Souza DC, Carmo DC, Santos JT, Branco E. Ramos colaterais do arco aórtico e suas principais ramificações no cachorro-do-mato (Cerdocyon thous). Pesq Vet Bras. 2016;36(7):647-51.

Lucherini M, Vidal EML. Lycalopex gymnocercus (Carnivora: Canidae). Mamm Species. 2008;820:1-9. 
Macedo BC, Lima AR, Pereira LC, Branco E. Descrição morfológica dos ramos colaterais da aorta abdominal do tamanduá-mirim (Tamandua tetradactyla). Biotemas. 2013;26(1):173-80.

Machado GV, Souza JR, Gonçalves PR, Parizzi A, Donin DG. A artéria celíaca e seus ramos no ratão-do-banhado (Myocastor coypus-Rodentia: Mammalia). Biotemas. 2002;15(2):41-54.

Machado MRF, Miglino MA, Cabral VP, Araújo N. Origem das artérias celíaca e mesentérica cranial em bubalinos (Bubalus bubalis, L. 1758). Braz J Vet Res Anim Sci. 2000;37(2):99-104.

Oliveira GB, Oliveira REM, Bezerra FVF, Câmara FV, Araújo Jr HN, Oliveira MF. Origem e distribuição da artéria celíaca em preás (Galea spixii Wagler, 1831). Cienc Anim Bras. 2017;18:e32918.

Pinheiro LL, Araújo EB, Lima AR, Martins DM, Melul R, Souza ACB, et al. The collateral branches from abdominal aorta in ocelot (Leopardus pardalis). Pesq Vet Bras. 2014b;34(5):491-5.

Pinheiro LL, Branco E, Souza DC, Pereira LHC, Lima AR. Descrição do plexo braquial do cachorro-do-mato (Cerdocyon thous Linnaeus, 1766). Cienc Anim Bras. 2014a;15(2):213-9.

Pinheiro LL, Lima AR, Souza ACB, Pereira LC, Branco ER. Topografia do cone medular do cachorro-do-mato (Cerdocyon thous Linnaeus, 1766): relato de caso. Biotemas. 2011;24(4):129-33.

Ramos AH, Santos LM, Miglino MA, Peres JA, Guerra RR. Biometria, histologia e morfometria do sistema digestório do cachorro-do-mato (Cerdocyon thous) de vida livre. Biotemas. 2011;24(4):111-9.

Roza MS, Marinho GC, Pereira JA, Salvador-Gomes M, Abidu-Figueiredo M. Celiac artery with a pulmonary branch in dog: a rare variation. J Morphol Sci. 2012;29(4):253-55.
Roza MS, Pestana FM, Fernandez JMF, Silva BX, AbiduFigueiredo M. Tronco celiaco-mesentérico em gato. Rev Port Cienc Vet. 2009;104(569-72):83-6.

Schummer A, Wilkens H, Vollmerhaus B, Habermehl $\mathrm{KH}$. The circulatory system, the skin, and the cutaneous organs of the domestic mammals. Berlin: Verlag Paul Parey; 1981. p. 1-268.

Sillero-Zubiri C, Hoffmann M, MacDonald DW (EE.). Canids: foxes, wolves, jackals and dogs. Cambridge, UK: IUCN - The World Conservation Union; 2004; p. 26-80.

Silva BX, Roza MS, Babinski MA, Scherer PO, Palhano HB, Figueiredo MA. Morfometria, origem e esqueletopia da artéria celíaca no gato doméstico. Rev Bras Med Vet. 2013;35(3):253-29.

Silva Bx, Roza MS, Babinski MA, Palhano HB, Scherer PO, Figueiredo MA. Ramos principais da artéria celíaca no gato doméstico: estudo aplicado à pesquisa experimental e a prática cirúrgica. R Bras Ci Vet. 2012; 19(3):133-8.

Souza Jr P, Carvalho NC, Mattos K, Santos ALQ. Origins and ramifications of brachial plexus in the crab-eatingfox Cerdocyon thous (Linnaeus, 1766). Pesq Vet Bras. 2014;34(10):1011-23.

Souza Jr P, Moraes FM, Carvalho NC, Canelo EA, Thiesen $\mathrm{R}$, Santos ALQ. Mandibular morphometry applied to anesthetic blockage in the maned wolf (Chrysocyon brachyurus). J Zoo Wildl Med. 2016;47(1):91-7.

Souza Jr P, Santos LMRP, Nogueira DMP, Abidu-Figueiredo M, Santos ALQ. Occurrence and morphometrics of the brachioradialis muscle in wild carnivorans (Carnivora: Caniformia, Feliformia). Zoologia. 2015;32(1):23-32.

Vaz MGR, Lima AR, Souza ACB, Pereira LC, Branco E. Estudo morfológico dos músculos do antebraço de cachorro-do-mato-de-orelhas-curtas (Atelocynus microtis) e cachorro-do-mato (Cerdocyon thous). Biotemas. 2011;24(4):121-7. 